\title{
Direct profiling of the phospholipid composition of adult Caenorhabditis elegans using whole-body imaging mass spectrometry
}

\author{
Saira Hameed $^{1}$ - Koji Ikegami ${ }^{1}$ - Eiji Sugiyama ${ }^{1}$ - Shoko Matsushita ${ }^{1}$. \\ Yoshishige Kimura $^{1}$ - Takahiro Hayasaka ${ }^{1,3}$ - Yuki Sugiura ${ }^{1,4}$ - Noritaka Masaki ${ }^{1}$. \\ Michihiko Waki ${ }^{1}$ - Isao Ohta ${ }^{2} \cdot$ Md Amir Hossen $^{1} \cdot$ Mitsutoshi Setou $^{1}$
}

Received: 14 April 2015 /Revised: 8 July 2015 / Accepted: 20 July 2015 / Published online: 27 August 2015

(C) The Author(s) 2015. This article is published with open access at Springerlink.com

\begin{abstract}
A protocol for the direct analysis of the phospholipid composition in the whole body of adult soil nematode, Caenorhabditis elegans (C. elegans), was developed, which combined freeze-cracking of the exoskeletal cuticle and matrix-assisted laser desorption/ionization-imaging mass spectrometry (MALDI-IMS). Biomolecules in the $m / z$ range from 700 to 900 were more effectively detected in the freezecracked than from simple frozen adult nematode bodies. Different distribution of biomolecules was observed in a nematode body when the matrix was applied with a sublimation deposition method. The whole-body IMS technique was applied on genetically deficient mutant $C$. elegans to combine whole-body lipidomics and genetics, by comparing the fatty
\end{abstract}

Published in the topical collection New Applications of Mass Spectrometry in Biomedicine with guest editors Fumio Nomura, Mitsutoshi Setou, and Toshimitsu Niwa.

Electronic supplementary material The online version of this article (doi:10.1007/s00216-015-8932-7) contains supplementary material, which is available to authorized users.

Mitsutoshi Setou

setou@hama-med.ac.jp

1 Department of Cell Biology and Anatomy, Hamamatsu University School of Medicine, 1-20-1 Handayama, Higashi-ku, Hamamatsu, Shizuoka 431-3192, Japan

2 Research Equipment Center, Hamamatsu University School of Medicine, 1-20-1 Handayama, Higashi-ku, Hamamatsu, Shizuoka 431-3192, Japan

3 Present address: Faculty of Health Sciences, Health Innovation and Technology Center, Hokkaido University, Kita 12 Nishi 5, Kita-ku, Sapporo, Hokkaido 060-0812, Japan

4 Present address: Department of Biochemistry, School of Medicine, Keio University, Shinjuku-ku, Tokyo 160-8582, Japan acid compositions, especially of the phosphatidylcholine (PC) species, between the wild-type and fat-1 mutants, which lack the gene encoding an $n-3$ fatty acid desaturase. A significant reduction of $\mathrm{PC}(20: 5 / 20: 5)$ and $\mathrm{PC}(20: 4 / 20: 5)$ and a marked increase of PC(20:4/20:4), PC(20:3/20:4), and $\mathrm{PC}(20: 3 / 20: 3)$ were detected in the fat- 1 mutants in positive ion mode. In addition, phospholipid compositions other than PCs were analyzed in negative ion mode. A loss of a possible phosphatidylinositol (PI) with 18:0/ $20: 5$ and a compensative accumulation of putative PI(18:0/20:4) were detected in the fat-1 mutants. In conclusion, the whole-body MALDI-IMS technique is useful for the profiling of multiple biomolecules in C. elegans in both intra- and inter-individual levels.

Keywords Caenorhabditis elegans · Cuticle · Exoskeleton · Freeze-cracking · Matrix-assisted laser desorption/ ionization-imaging mass spectrometry $\cdot$ Phosphatidylcholine . Phosphatidylinositol

\section{Introduction}

Recent advances in mass spectrometry have enabled the direct analyses of biomolecules in tissue samples without any targetspecific labeling [1, 2]. Matrix-assisted laser desorption/ ionization-imaging mass spectrometry (MALDI-IMS) can be used for the analyses of the spatial distribution of various biomolecules, which range from small metabolites to lipids, peptides, and intact proteins, in tissue sections [3-6]. The state of the art MALDI-IMS technique has been used for the investigation of molecular distributions in mammalian tissues [7], including samples of diseased human tissues [8-10]. It has also been used for label-free non-targeted analyses of 
biomolecules in various species [11], such as microbes [12], plants [13], parasites [14], arthropods, including crustaceans such as the giant tiger prawn (Penaeus monodon) [15], and insects such as the fruit fly (Drosophila melanogaster) [16, 17].

The soil nematode, Caenorhabditis elegans (C. elegans), is a common model organism and is extensively used in life science research [18]. C. elegans has a wide range of advantages for experimental research [19]. The short life span of minimally 3 days and the capability of being frozen enable a number of iteration of experimental tests in a short time [18]. These allow quick forward genetics, combined with the ease of genetic manipulations [20]. The body structure with multiple organs composed of the fixed number of cells $(\sim 1000$ somatic cells) [18], with well-characterized cell fate is highly powerful to developmental biology. Given these advantages in multiple directions, C. elegans has a big potential to provide a powerful platform for "Integrating-Omics," in which genetics, transcriptomics, proteomics, lipidomics, and metabolomics are combined to find new insights [21], opening a new era of life sciences. C. elegans also begins to be used in applied sciences, such as drug discovery or screening [22].

Some studies have investigated the metabolomic profiling of genetically deficient mutant nematodes [23-28]. C. elegans has a balloon-like body with high osmotic pressure that is enclosed by an exoskeleton consisting of a tough impermeable cuticle [29]. This has hindered the direct detection and analysis of the biomolecules contained inside. Thus, the components of nematode bodies have been extracted in most metabolomics analyses. The very thick and rigid exoskeleton was thought to inhibit a direct "whole-body" IMS, as the cuticle layer of plants should be bypassed using vibratome sectioning before the IMS analysis of molecules beneath their cuticles [30]. Moreover, the cryosectioning of exoskeletal organisms requires special handling, which is time-consuming and requires well-designed equipment [16]. Thus, the development of a facile sample preparation for the analysis of the biomolecules using the whole-body IMS of $C$. elegans has been desired.

Our aim was to establish a facile protocol for the wholebody MALDI-IMS of adult $C$. elegans. To accomplish this, we combined a freeze-cracking technique with MALDI-IMS and were able to successfully analyze nematodes to visualize biomolecules in an individual nematode level. We further combined the whole-body MALDI-IMS to genetics through the comparison of the wild-type and fat-1 mutants and succeeded in detecting significant differences in the fatty acid compositions of the phosphatidylcholine (PC) and phosphatidylinositol (PI) species between the two genetically different nematode lines.

\section{Materials and methods}

\section{Chemicals}

Methanol $(\mathrm{MeOH})$, ethanol (EtOH), chloroform $\left(\mathrm{CHCl}_{3}\right)$, ultrapure water, and potassium acetate $\left(\mathrm{CH}_{3} \mathrm{COOK}\right)$ were purchased from Wako Pure Chemical Industries (Osaka, Japan). Calibration standard peptides (human bradykinin and angiotensin II) were purchased from Bruker Daltonics (Billerica, MA, USA). 2,5-Dihydroxybenzoic acid (DHB) was purchased from Bruker Daltonics (Billerica, MA, USA) or Sigma-Aldrich (St. Louis, MO, USA). 9-Aminoacridine hemihydrate (9-AA) was purchased from Acros Organics (NJ, USA).

\section{Nematodes}

C. elegans strains were grown at $20^{\circ} \mathrm{C}$ under standard conditions [18] on nematode growth medium (NGM) agar plates $(0.3 \% \mathrm{NaCl}, 0.25 \%$ Bacto Peptone, $1.5 \%$ agar, $0.0005 \%$

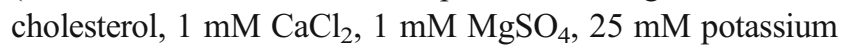
phosphate buffer [ $\mathrm{pH} 6.0]$ ), which were seeded with the OP50 Escherichia coli strain as a food source. The wild-type strain (Bristol N2) and fat-1 mutant (BX24: fat-1(wa9) IV) of C. elegans were obtained from the Caenorhabditis Genetics Center (Minneapolis, MN, USA). A synchronous culture of C. elegans was obtained by bleaching the nematodes 3 days before observation. Only adult nematodes were used for the MALDI-IMS and liquid chromatography-electrospray ionization-tandem mass spectrometry (LC-ESI-MS/MS) analyses.

\section{Sample preparation for MALDI-IMS}

The nematodes that were grown on the surface of the NGM agar plates were harvested by thoroughly washing with water (Fig. 1a), and the live nematodes were transferred into a glass tube using a Pasteur pipette (Fig. 1b). Subsequently, water droplets containing live nematodes were transferred onto indiumtin-oxide (ITO)-coated glass slides (Bruker Daltonics) (Fig. 1c). The freeze-cracking method involved covering the nematode containing water droplets with a cover glass that was lightly pressed with a finger to immobilize the nematodes and then to make direct contact with their surfaces (Fig. 1d). The sample slide was then rapidly frozen on an aluminum block at liquid nitrogen temperature $\left(-196^{\circ} \mathrm{C}\right)$ (Fig. 1e). The aluminum block allowed for a faster heat transfer, which generated a temperature gradient across $C$. elegans because of their cylindrical morphology. The cover glass was quickly detached to remove the cracked cuticle exoskeletons (Fig. 1f). The sample slide was then dried under vacuum for $\sim 1 \mathrm{~h}$ (Fig. 1g).

To compare the freeze-cracked and simple frozen nematodes, water droplets containing live nematodes were transferred to the left and right sides of an ITO-coated glass slide. The left side was used for the simple frozen nematodes, 
Fig. 1 Workflow for whole-body MALDI-IMS analyses of adult C. elegans. a, b Harvesting live nematodes from a nematode growth medium (NGM) agar plate into a glass tube by suspension in water. $\mathbf{c}$ Seeding the nematodes onto an ITO-coated glass slide. d Applying a cover glass on a nematode subset and pressing them for freeze-cracking (on the right side of the glass slide). The other subset of live nematodes was left untreated. e Rapid freezing of the nematodes on a liquid nitrogen-cooled aluminum block. Through this process, the nematodes that were covered by the coverslip were freeze-cracked, whereas the others were simply frozen. $\mathbf{f}$ Removing the coverslip to detach the cuticle exoskeleton from the nematodes. $g$ Vacuum drying of the nematodes on a pre-cooled aluminum block

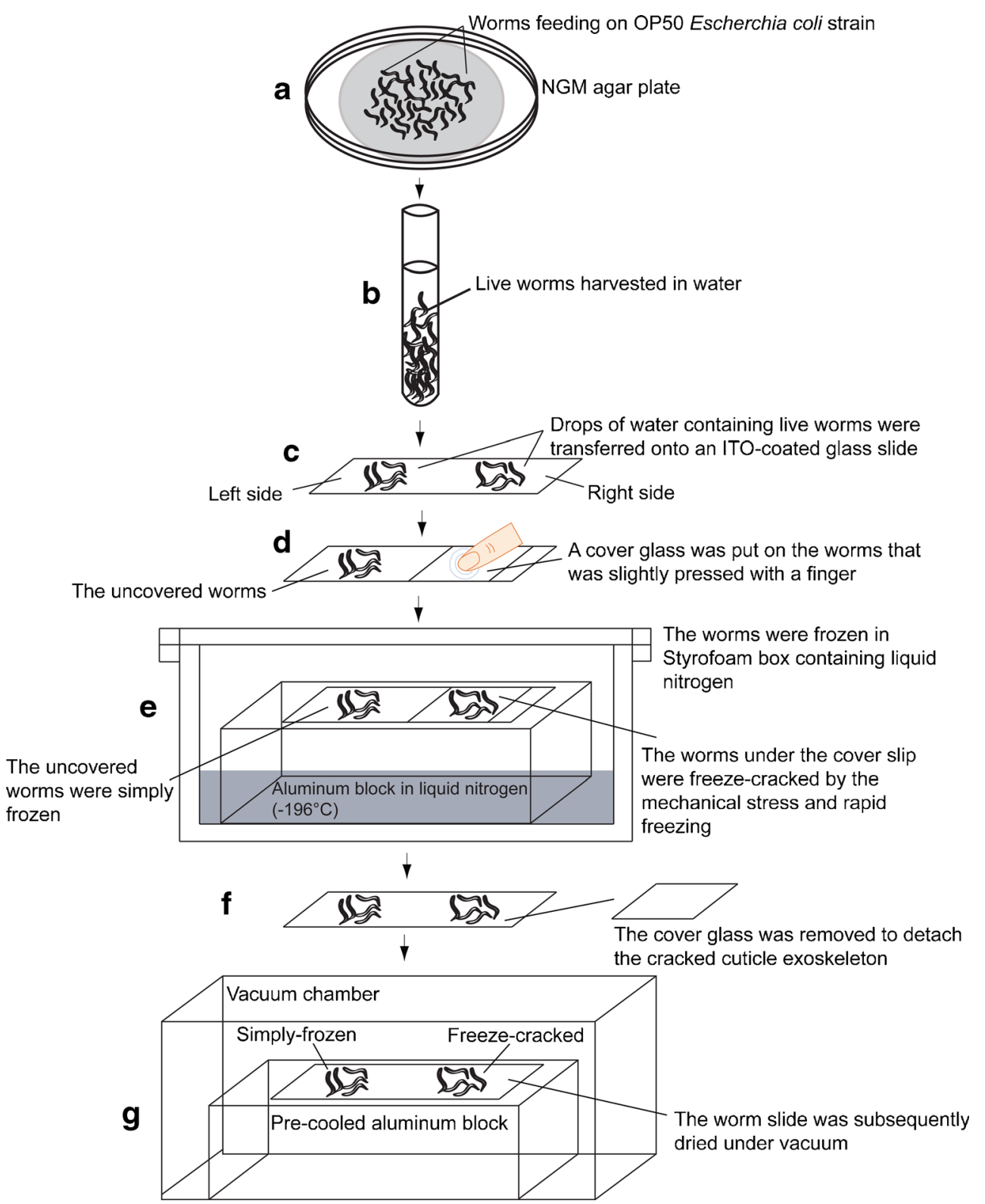

whereas the right was used for the freeze-cracked nematodes (Fig. 1d). Simple frozen nematodes were not subjected to any cracking treatment but were frozen and dried simultaneously with cracked ones (Fig. 1e-g).

\section{Scanning electron microscopy}

Scanning electron microscopy (SEM) was performed as previously reported [31, 32]. Simple frozen and freezecracked nematode specimens were coated with osmium tetroxide $\left(\mathrm{OsO}_{4}\right)$ using a plasma multi coater model PMC-5000 (Meiwa, Japan). The SEM observation of nematodes was performed using a Hitachi S-4800 field emission scanning electron microscope at an acceleration voltage of $1.0 \mathrm{kV}$ and an emission current of $10 \mu \mathrm{A}$. The vacuum level in the observation chamber was $10^{-5}$ $10^{-7} \mathrm{~Pa}$, and the working distance was $8.0 \mathrm{~mm}$.

\section{Matrix application}

DHB was chosen to detect PC in positive ion mode, since it is most commonly used for MALDI-MS and MALDI-IMS with high vacuum chamber [33-35]. 9AA was used to detect PI in negative ion mode, since it is most common for lipid analyses in negative ion mode of MALDI-MS and MALDI-IMS [36, 37]. To extract and co-crystallize the analytes, the nematode samples were spray-coated with DHB solution $(50 \mathrm{mg} / \mathrm{mL}$ in $70 \% \mathrm{MeOH}$ containing $20 \mathrm{mM} \mathrm{CH} \mathrm{CH}_{3} \mathrm{COOK}$ ) for analyses in positive ion mode, or 9 -AA solution $(10 \mathrm{mg} / \mathrm{mL}$ in $70 \% \mathrm{EtOH})$ 
for analyses in negative ion mode on an ITO-coated glass slide using a 0.2-mm caliber nozzle airbrush (Procon Boy FWA Platinum; Mr. Hobby, Tokyo, Japan). Approximately $2 \mathrm{~mL}$ of DHB or $3 \mathrm{~mL}$ of 9-AA solutions were sprayed over 15 to $20 \mathrm{~min}$. The airbrush was moved right to left, top to bottom, and vice versa, for around 1200 times, while maintaining a distance of $10 \mathrm{~cm}$ between the nozzle and nematode tissue surfaces. After the matrix application, the slide was incubated in desiccator for around $5 \mathrm{~min}$. The humidity of room was maintained under $25 \%$ at $23{ }^{\circ} \mathrm{C}$. The glass slide was observed by a microscope to confirm whether the matrix layer uniformly covered the nematode sample surfaces.

The nematode samples were also subjected to matrix application by sublimation deposition method, with $600 \mathrm{mg}$ of DHB sublimated at $170{ }^{\circ} \mathrm{C}$ for the deposition thickness to reach $1.5 \mu \mathrm{m}$, using iMLayer (Shimadzu, Japan).

\section{MALDI-IMS}

MALDI-IMS was performed using an ultraflex II TOF/TOF instrument (Bruker Daltonics), equipped with a Smartbeam-II $\mathrm{Nd}$ :YAG $355 \mathrm{~nm}$ laser, with $25 \mu \mathrm{m}$ in raster scan pitch. The laser frequency was $200 \mathrm{~Hz}$, and the data were acquired using an ion source voltage of $25 \mathrm{kV}$ and a reflector voltage of $26.30 \mathrm{kV}$ in the positive ion reflectron mode. Calibration of the MS was performed using DHB $\left([\mathrm{M}+\mathrm{H}]^{+}, m / z\right.$ 155.03), human bradykinin fragment $1-7\left([\mathrm{M}+\mathrm{H}]^{+}, m / z 757.40\right)$, and human angiotensin II $\left([\mathrm{M}+\mathrm{H}]^{+}, m / z\right.$ 1046.54). In negative ion mode, the laser frequency was $100 \mathrm{~Hz}$, and the data were acquired using an ion source voltage of $20.11 \mathrm{kV}$ and a reflector voltage of $21.07 \mathrm{kV}$. The data acquisition areas, over which the spectra were measured, were set by tracing the outline of the well-cracked nematodes. These were characterized by the observation of the nematode bodies and the region immediately outside them. The mass spectra were acquired by averaging the signals from 500 laser pulses per sample measurement point, and the mass measurement range was set to $\mathrm{m} / \mathrm{z}$ 700-1000. The nematode specimens were automatically raster scanned using flexControl (ver. 3 or 3.4) and flexImaging (ver. 2.1 or 4.0) software (Bruker Daltonics). The acquired raw mass spectra were normalized to the total ion current (TIC). The images of the detected molecular ions were constructed using the flexImaging (2.1 and 4.0) software. Six simple frozen and six freeze-cracked nematodes were analyzed to evaluate the effectiveness of the exoskeleton removal. Four nematodes were analyzed to examine whether biomolecules were retained in nematode bodies. Six wild-type and fat1 mutant nematodes were analyzed in positive ion mode. Eight wild-type and fat-1 mutant nematodes were analyzed in negative ion mode. The data were presented as plots for each nematode and the mean of the signal intensities acquired from the multiple samples.
Table 1 Molecular weights of the phosphatidylcholine species in C. elegans

\begin{tabular}{lllll}
\hline PC species & MW & $\mathrm{H}^{+}$Adduct & $\mathrm{K}^{+}$Adduct & ID \\
\hline PC(20:5/20:5) & 825.5 & 826.5 & 864.5 & HMDB08511 \\
PC(20:4/20:5) & 827.5 & 828.5 & 866.5 & HMDB08478 \\
PC(20:4/20:4) & 829.5 & 830.5 & 868.5 & HMDB08476 \\
PC(20:3/20:4) & 831.5 & 832.5 & 870.5 & HMDB08379 \\
PC(20:3/20:3) & 833.5 & 834.5 & 872.5 & HMDB08377 \\
\hline
\end{tabular}

http://www.hmdb.ca/spectra/ms/search

\section{Liquid chromatography-electrospray ionization-tandem mass spectrometry (LC-ESI-MS/MS)}

Phospholipids of the harvested wild-type and fat-1 mutants were extracted with Folch method [38], and they were analyzed using LC-ESI-MS/MS with a 4000QTRAP triple quadrupole linear ion trap mass spectrometer (AB SCIEX, Framingham, MA, USA) equipped with an ACQUITY ultra-performance liquid chromatography system (Waters, Milford, MA, USA). An ACQUITY UPLC BEH C18 column $(2.1 \times 50 \mathrm{~mm}$, i.d., $1.7 \mathrm{~mm}$ particles; Waters $)$ was connected to a guard column $(2.1 \times 5 \mathrm{~mm}$; Waters), and the temperature of column oven was maintained at $40{ }^{\circ} \mathrm{C}$ [9]. The mobile phase consisted of a gradient of two solvent mixtures. Solvent A was composed of acetonitrile, $\mathrm{MeOH}$, and water (19:19:2 v/v/v), containing formic acid (0.1 vol.\%) and ammonia (0.028 vol.\%). Solvent $\mathrm{B}$ was composed of isopropanol containing formic acid (0.1 vol.\%) and ammonia (0.028 vol.\%). A gradient elution using solvents $\mathrm{A}$ and $\mathrm{B}$ was performed at a flow rate of $0.40 \mathrm{~mL} / \mathrm{min}$. To profile the molecular species of the specific phospholipid classes, a precursor ion scanning for the polar head groups of the PCs and sphingomyelins (SMs) $(m / z=184)$ was performed using the positive ion detection mode of the 4000Q-TRAP instrument. Fragment ions were generated through collision-induceddissociation (CID) [39]. The optimal collision energy was determined by the preliminarily analysis of PC(16:0/18:1), which was used as a standard lipid. The $\mathrm{PC}$ molecular species detected were assigned using their

Fig. 2 Freeze-cracked nematode bodies yield stronger signals. a Scanning electron microscopy images of simple frozen and freezecracked C. elegans. Scale bars: $200 \mu \mathrm{m}$ in low magnification (top), $25 \mu \mathrm{m}$ in high magnification (bottom). b, c Averaged mass spectra ranging from $\mathrm{m} / \mathrm{z} 700$ to 1000 that were detected in the whole body of b simple frozen and $\mathbf{c}$ freeze-cracked C. elegans. Four of the major mass peaks at $m / z 796.5,806.5,846.6$, and 868.5 were selected to depict the thermal color scale images. d Optical images and thermal color scale images of the four selected molecules on the nematode bodies. Scale bar: $200 \mu \mathrm{m}$. Color scale: deep blue, faint signal; red-purple, maximum signal. e Quantitative signal intensities on nematode bodies. The data are shown as plots for each nematode (diamonds; $n=6$ ) and mean (red line) \pm $\mathrm{SD}$ (blue lines). The $p$ values were calculated using a $t$ test 
$\mathrm{m} / \mathrm{z}$ values and the relative retention times of PC molecular species that were previously described $[9,34,39]$, or by referring to the online database, "The Human
Metabolome Database (HMDB)" (http://www.hmdb.ca/ spectra/ms/search), and the total fatty acid composition of C. elegans [23-25] (Table 1).

a
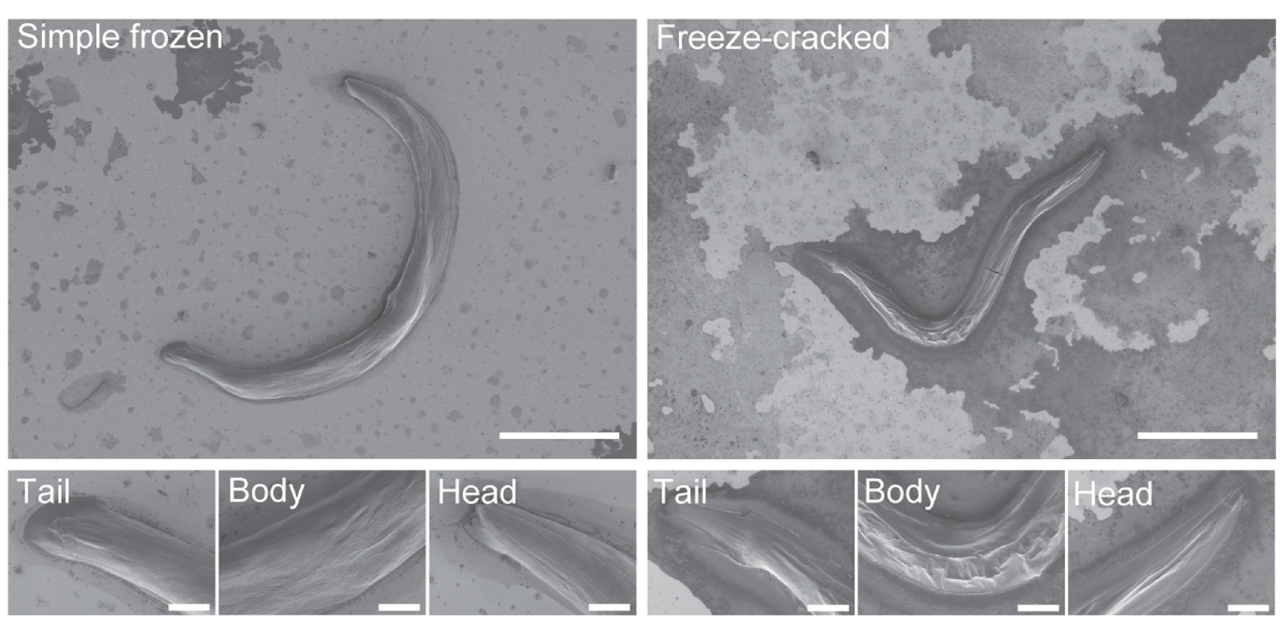

b
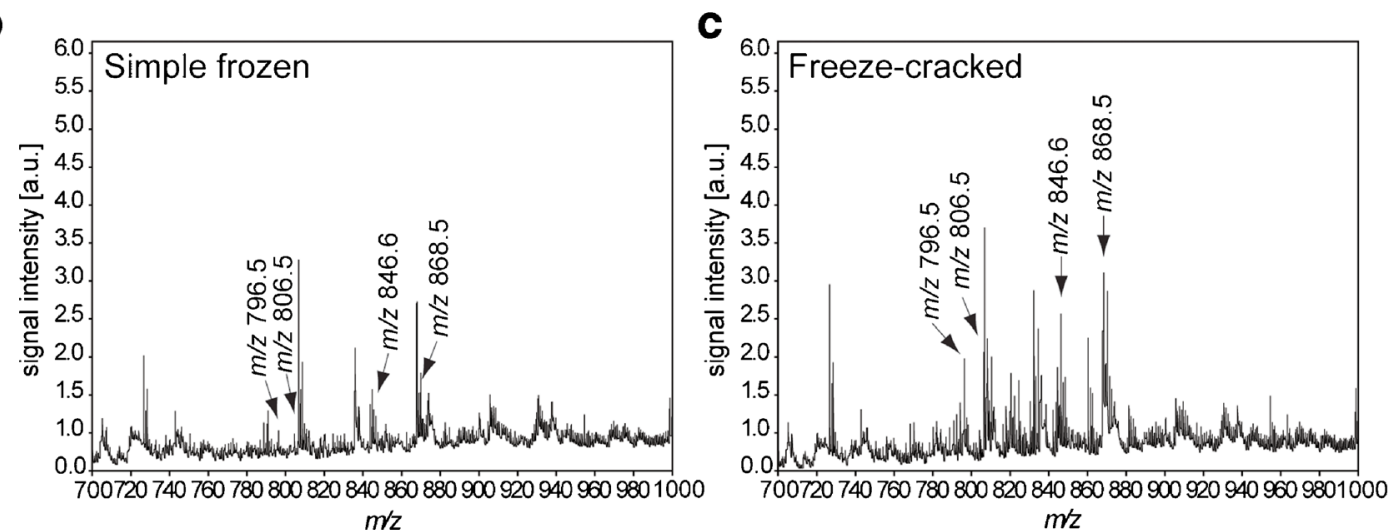

d $m / z$

$m z$
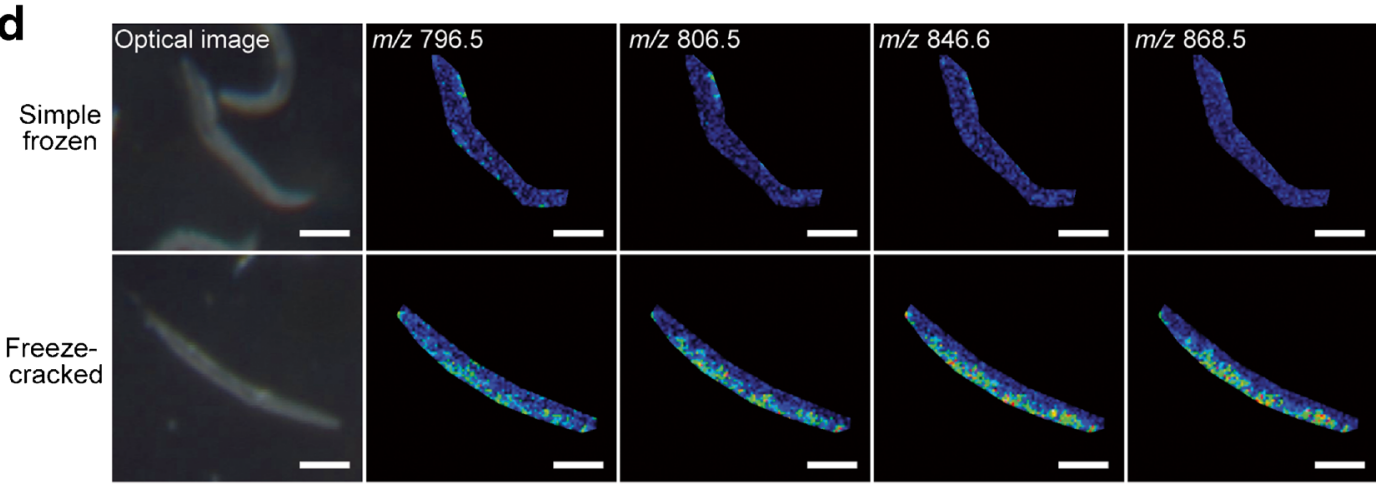

e
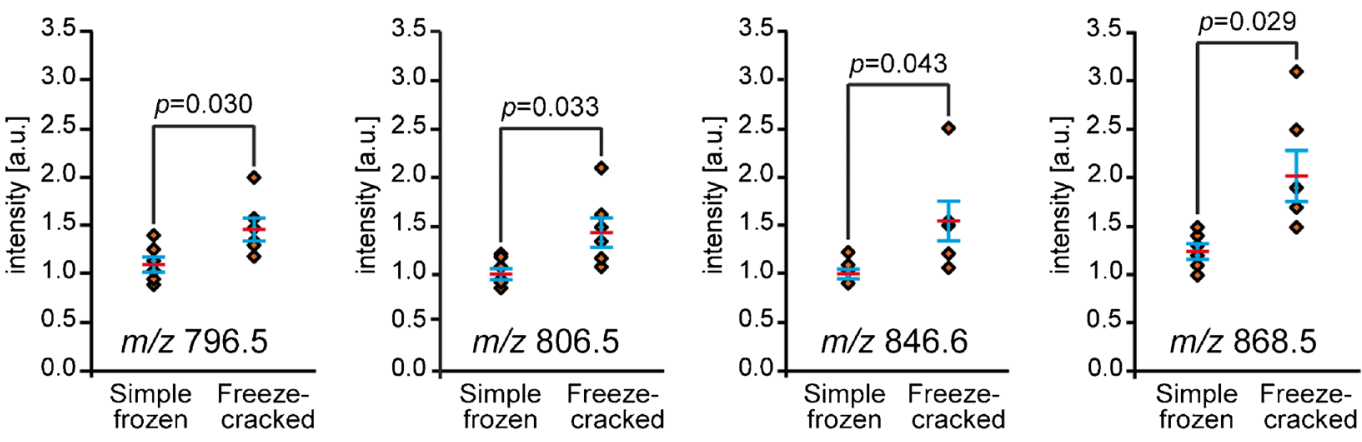
Further analyses of fatty acid compositions in the PC species of interest were performed by a hybrid quadrupoleOrbitrap mass spectrometer (Q Exactive; Thermo Scientific, Waltham, MA, USA), with the mass resolution of $5 \mathrm{ppm}$. Separation of the molecular species was carried out using Agilent1100 series HPLC System (Agilent Technologies, Germany) equipped with Acclaim ${ }^{\mathrm{TM}} 120 \mathrm{C} 18$ column (2.1 $\times$ $150 \mathrm{~mm}$, i.d., $3 \mu \mathrm{m}$ particles; Thermo Scientific). The temperature of the column oven was maintained at $50{ }^{\circ} \mathrm{C}$. The injection volume was $5.0 \mu \mathrm{L}$. The temperature of the sample tray was kept at $10^{\circ} \mathrm{C}$. Solvent A was composed of water, acetonitrile, $\mathrm{MeOH}(2: 1: 1, v / v / v)$, containing ammonium formate $(5 \mathrm{mM})$ and formic acid $(0.1$ vol.\%). Solvent B was composed of acetonitrile, isopropanol $(1: 9, v / v)$, containing ammonium formate $(5 \mathrm{mM})$ and formic acid $(0.1$ vol.\%). A gradient elution using solvents $\mathrm{A}$ and $\mathrm{B}$ was performed at a flow rate of $0.30 \mathrm{~mL} / \mathrm{min}$ for $50 \mathrm{~min}$ from the initial composition (A/B: $80 / 20, v / v)$ to the final composition (A/B: 0/100 vol.\%) with a linear gradient. MS and MS/MS analyses were performed in both positive and negative ion modes. MS spectra were acquired in the range of $m / z 700-900$. MS/MS spectra near the peak top of interested peaks were acquired with a targeted MS/ MS method. The target mass-resolving power at $\mathrm{m} / \mathrm{z} 200$ was set to 70,000 for both MS and MS/MS analyses. The isolation window for MS/MS was set to $0.4 \mathrm{~m} / \mathrm{z}$. The temperature of ion source heater was set to $350{ }^{\circ} \mathrm{C}$, and the capillary temperature was at $250{ }^{\circ} \mathrm{C}$. The ion spray voltage was set to $3.5 \mathrm{kV}$ for both ion modes. Maximum injection time was set to $100 \mathrm{~ms}$ for both MS and MS/MS analyses. The automatic gain control target was set to $1 \times 10^{7}$ for MS and $2 \times 10^{5}$ for MS/MS. The normalized collision energy for MS/MS was set to $30 \%$. Extracted ion chromatograms (EICs) were generated within a theoretical value of $\pm 5 \mathrm{ppm}$ for PC species of interest.

\section{Results}

\section{Relative effectiveness of the freeze-cracking method in the direct detection of multiple biomolecules}

To evaluate the effectiveness of the exoskeleton removal, we performed the comparative analyses of the two procedures, simple freezing and freeze-cracking, in parallel on a glass slide (Fig. 1). To effectively remove the exoskeletal cuticle of C. elegans and expose the internal structures of the nematodes, we placed a cover slip on the nematodes and pressed it with a finger before chilling them using a liquid nitrogencooled aluminum block (Fig. 1d). We evaluated the effectiveness of this procedure by comparing it with a simple freezing method. We observed the condition of the samples using an SEM. The surface of the freeze-cracked nematode bodies looked highly scabrous with multiple wrinkles (Fig. 2a; right), whereas the simple frozen nematodes had a highly smooth surface, which appeared to retain an intact exoskeletal cuticle (Fig. 2a; left). The microscopy showed that freeze-cracking drastically changed the surface condition of the sample specimens.

We then analyzed the freeze-cracked nematodes using MALDI-IMS and compared the signal intensity of the biomolecules with those from the simple frozen nematodes (Fig. 2b, c). The averaged mass spectra obtained from the freezecracked nematodes had increased signal intensity throughout the $m / z$ range 700-1000 when compared to the simple frozen nematodes (Fig. 2b vs. Fig. 2c). In particular, the molecular ions that were observed between $\mathrm{m} / \mathrm{z} 700$ and 900 were detected with much higher signal intensities from the freezecracked nematodes than those from the simple frozen nematodes. We selected four molecules with $\mathrm{m} / \mathrm{z} 796.5,806.5$, 846.6, and 868.5, respectively (arrows in Fig. 2b, c), to visually compare their signal intensities from the nematode bodies. The signal intensities of these selected molecules increased by $>50 \%$ of the maximum signal level (yellow-to-red colors) in some regions of freeze-cracked nematodes, whereas those in the simple frozen nematodes appeared near the noise level (blue-to-cyan colors) (Fig. 2d). We performed further semiquantitative analyses of the signal intensities of the four molecules. In all four molecules, the signal intensities detected from the freeze-cracked nematodes were significantly higher than those from the simple frozen nematodes $(n=6)$ (Fig. 2e). These results demonstrated that the freeze-cracking method enabled the highly effective direct detection of multiple internal biomolecules, which provided a facile whole-body MALDI-IMS.

\section{Molecular distribution analyses in nematode bodies by combining the freeze-cracking method with the matrix sublimation}

The nematodes were pressed with a finger during the freeze-cracking procedure (Fig. 1d). This has a potential risk in that the nematode bodies could be punctured during this process, which could result in the delocalization or drift of intra-body ingredients from the nematode body to the surrounding area. Thus, we evaluated whether the biomolecules were retained in the nematode bodies. For this evaluation, we expanded the imaging measurement areas to include the glass surface adjacent to the nematode bodies (Fig. 3a, b; insets) and compared the mass spectra from regions of interest (ROIs) inside and outside of the nematode bodies (Fig. 3a, b; insets). The averaged mass spectrum acquired from the ROI inside of the nematode body (Fig. 3a) has several mass peaks with $\mathrm{m} / \mathrm{z}$ values ranging from 750 to 900 , whereas that taken from the ROI outside of the nematode body had few significant mass peaks (Fig. 3b). 


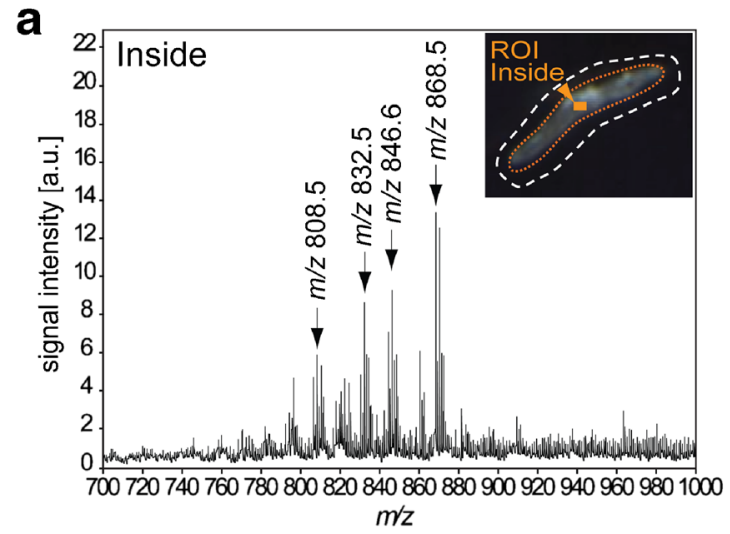

C
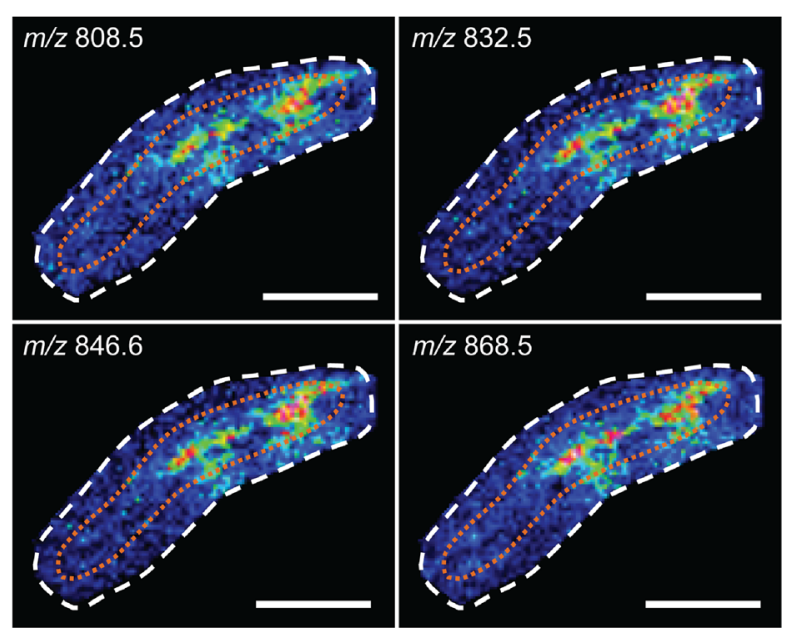
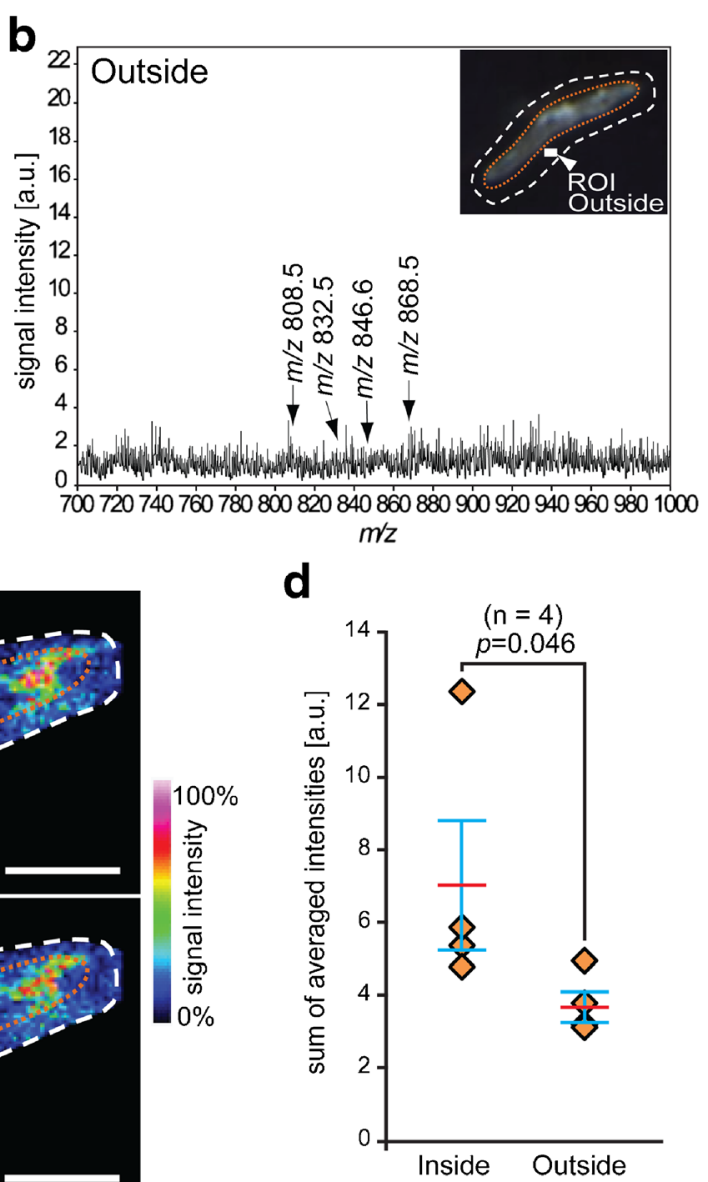

e

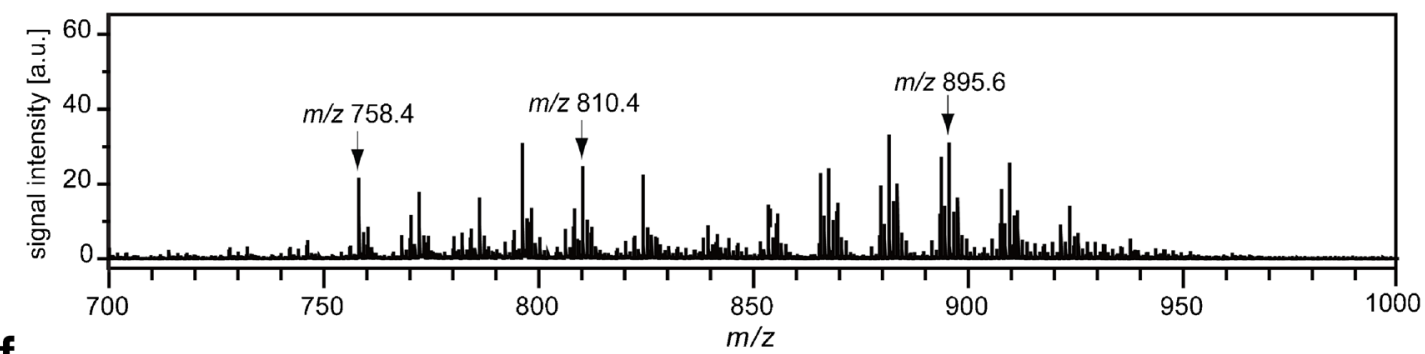

f
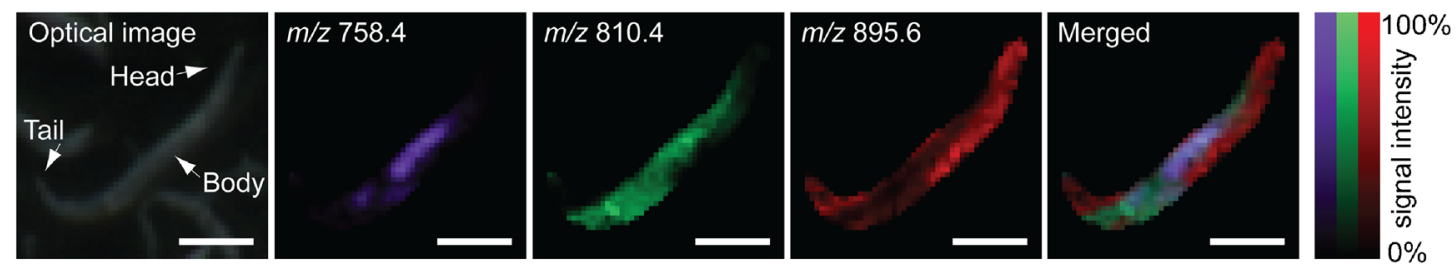

Fig. 3 Freeze-cracking method allows analyses of molecular distribution in the nematode body. a, b Averaged mass spectra detected from the regions of interest $\mathbf{a}$ inside and $\mathbf{b}$ outside of the nematode body. Four of the major mass peaks at $\mathrm{m} / \mathrm{z} 808.5, \mathrm{~m} / \mathrm{z} 832.5, \mathrm{~m} / \mathrm{z} 846.6$, and $\mathrm{m} / \mathrm{z} 868.5$ were selected to depict thermal color scale images. Insets show the outline of nematode body (orange dotted line) and scanned area in IMS (white dotted line). $\mathbf{c}$ Thermal color scale images of the selected four molecules. Scale bar: $250 \mu \mathrm{m}$. Color scale: deep blue, faint signal; red-purple,

maximal signal. d Quantitative signal intensities in the regions of interest. The data are shown as plots for each nematode (diamonds; $n=$ 4 ) and mean (red line) $\pm \mathrm{SD}$ (blue lines). The $p$ values were calculated using a paired $t$ test. e An averaged mass spectrum detected from freezecracked nematode body with DHB matrix applied by sublimation. Three of major mass peaks at $\mathrm{m} / \mathrm{z} 758.4,810.4$, and 895.6 were selected to depict pseudo color scale images. f Pseudo color scale images of the selected three molecules. Scale bar: $250 \mu \mathrm{m}$ 
We selected four of major ion peaks, $\mathrm{m} / \mathrm{z}$ values of 808.5 , 832.5, 846.6, and 868.5 (arrows in Fig. 3a, b), to monitor whether or not those effectively detectable molecules are drifted from the worm body to the outside of body. All four of the molecular ions showed signal intensities that increased by $>50 \%$ of the maximum from the inside area of the nematode body (yellow-to-red colors), whereas they appeared near the background level in the area outside of nematode body (blue-to-cyan colors) albeit slightly delocalized to the surrounding glass surface (Fig. 3c). The semi-quantitative analyses of nematodes demonstrated that the averaged signal intensities of all four of the molecules were significantly higher inside than outside the nematode bodies $(n=4)$ (Fig. 3d). These results indicated that the biomolecules were retained inside nematode bodies during the freeze-cracking process.

We further sought to analyze the distribution of biomolecules inside the nematode body with matrix applied via a sublimation deposition method. The averaged mass spectrum acquired from nematode bodies provided a number of mass peaks (Fig. 3e). We selected three of major mass peaks at $\mathrm{m} / \mathrm{z}$ $758.4, \mathrm{~m} / \mathrm{z} 810.4$, and $\mathrm{m} / \mathrm{z} 895.6$ to observe the spatial distribution of those molecules in the nematode body. These three molecules showed clearly different distribution patterns in the nematode body: the molecule with $m / z 758.4$ was strongly localized at the main body trunk (Fig. 3f; purple); the molecule with $\mathrm{m} / \mathrm{z} 810.4$ was detected almost throughout the nematode body, except in the head and tail regions (Fig. 3f; green); the molecule with $\mathrm{m} / \mathrm{z} 895.6$ was strongly detected in the head and tail regions (Fig. 3f; red).

\section{Comparative analyses of fatty acid composition of phosphatidylcholine in wild-type and genetically deficient nematodes}

We sought to evaluate the potential of our technique for use in combination with nematode genetics for general versatility in analytical biochemistry. For this, we tested the fatty acid composition of PC in the wild-type and genetically deficient fat-1 mutant nematodes. The fat-1 mutants lack the gene encoding an $n-3$ fatty acid desaturase [23]. Before analyzing the fat- 1 mutants using whole-body MALDI-IMS, we extracted their lipids and profiled the total PC compositions of the two nematode strains by precursor ion scan of $m / z 184$, which corresponds to the head group of PC and SM, using LC-ESI-MS/ MS. We focused on PC species that contain fatty acids reported to be drastically altered in the fat 1 mutant (Fig. 4a; red and green) [23], i.e., $\operatorname{PC}(20: 5 / 20: 5), \operatorname{PC}(20: 4 / 20: 5), \operatorname{PC}(20: 4 /$ $20: 4), \operatorname{PC}(20: 3 / 20: 4)$, and $\operatorname{PC}(20: 3 / 20: 3)$. Figure $4 b$ shows a $\mathrm{PC}$ ion intensity map ( $x$-axis: retention time, $y$-axis: $\mathrm{m} / z$ ), in which the PC ion "spots" from the wild-type and fat-1 mutants were colored red and green, respectively. This merged contour plot showed a clear difference in the PC composition between them (Fig. 4b). Signals at $\mathrm{m} / \mathrm{z} 828.5$ and $\mathrm{m} / \mathrm{z} 826.5$,
Fig. 4 Fatty acid composition of phosphatidylcholine in fat-1 mutant. a Fatty acid desaturation pathway modified from Watts and Browse [23]. Red: exclusively detected in the wild type [23]. Green: increased in fat-1 mutants [23]. $S A$ stearic acid, $O A$ oleic acid, $L A$ linoleic acid, $A L A$ alphalinolenic acid, GLA gamma-linolenic acid, STA stearidonic acid, DGLA dihomo-gamma-linolenic acid, O3AA omega-3 arachidonic acid, $A A$ arachidonic acid, EPA eicosapentaenoic acid. b Merged contour plot obtained by precursor ion scanning of $m / z 184$ on lipid extracts from WT and fat-1: WT (red), fat-1 mutant (green), both (yellow). c Merged EICs (left column): wild-type (red) and fat-1 mutant (green). MS/MS spectra at near peak top of picked peaks acquired by targeted MS/MS: In positive ion mode, $[\mathrm{M}+\mathrm{H}]^{+}$ions produced a common fragment ion at $\mathrm{m} / \mathrm{z} 184.074$ [phosphocholine $+\mathrm{H}]^{+}$(middle panel). In negative ion mode, $[\mathrm{M}+\mathrm{HCOO}]^{-}$ions produced fragment ions depending on their fatty acid composition (right panel)

corresponding to $[\mathrm{PC}(40: 9)+\mathrm{H}]^{+}$and $[\mathrm{PC}(40: 10)+\mathrm{H}]^{+}$, were almost exclusively detected in wild-type nematodes (Fig. 4b; red). In contrast, signals at $\mathrm{m} / \mathrm{z} 834.5$ and $\mathrm{m} / \mathrm{z} 832.5$, corresponding to $[\mathrm{PC}(40: 6)+\mathrm{H}]^{+}$and $[\mathrm{PC}(40: 7)+\mathrm{H}]^{+}$, were selectively detected in the fat-1 mutants (Fig. 4b; green). A signal of $m / z 830.5$ corresponding to $[\mathrm{PC}(40: 8)+\mathrm{H}]^{+}$was detected in comparable quantities in both strains (Fig. 4b; yellow).

We performed further LC-ESI-MS/MS analyses with a hybrid quadrupole-Orbitrap mass spectrometer to determine precise fatty acid compositions of the PC species. PC species were detected as $[\mathrm{M}+\mathrm{H}]^{+}$in positive ion mode and $[\mathrm{M}+\mathrm{HCOO}]^{-}$in negative ion mode [40]. Figure 4c (left column) shows merged EICs of wild-type (red) and fat- 1 mutant (green) in positive ion mode. EICs corresponding to $[\mathrm{M}+\mathrm{HCOO}]^{-}$of each $\mathrm{PC}$ species showed almost the same pattern as that of $[\mathrm{M}+\mathrm{H}]^{+}$(see Electronic Supplementary Material (ESM) Fig. S1). MS/MS spectra of the most major peak in EICs at each $\mathrm{m} / \mathrm{z}$ in the two strains were obtained (Fig. 4c; middle and right columns). MS/MS spectra of minor peaks in EICs were shown in ESM Fig. S2. Consistently with the result of the precursor ion scan, the peaks at $\mathrm{m} / \mathrm{z} 834.6$ (36.6 $\mathrm{min}$ ), $\mathrm{m} / \mathrm{z} 832.6$ (35.7 $\mathrm{min}$ ), $\mathrm{m} / \mathrm{z} 830.6$ (34.6 $\mathrm{min}), \mathrm{m} / \mathrm{z} 828.6$ (32.8 $\mathrm{min}$ ), and $\mathrm{m} / \mathrm{z} 826.5$ (31.6 $\mathrm{min}$ ) in positive ion mode produced a common fragment ion at $\mathrm{m} / \mathrm{z}$ 184.0736, which corresponds to [phosphocholine $+\mathrm{H}]^{+}$ (Fig. 4c; middle column). MS/MS spectra of negative ion mode showed fragment ion peaks that corresponded to fatty acids (Fig. 4c; right column). PC species containing eicosapentaenoic acid (EPA), PC(20:5/20:5) and PC(20:4/20:5), were almost exclusively detected in wild-type nematodes (Fig. 4c). In contrast, PC species that did not contain EPA, PC(20:4/20:4), PC(20:3/ $20: 4)$, and $P C(20: 3 / 20: 3)$, were detected much more strongly in the fat-1 mutants than wild type (Fig. 4c).

\section{Comparative whole-body MALDI-IMS analyses of phospholipid composition in wild-type and genetically deficient nematodes}

With the identified information for the fatty acid compositions of PC molecules in the wild-type and fat-1 mutants, we 
a

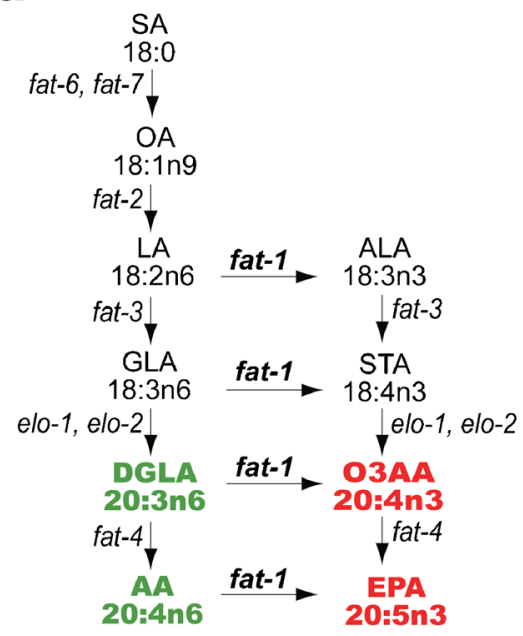

fat-1: $n-3$ fatty acid desaturase

C

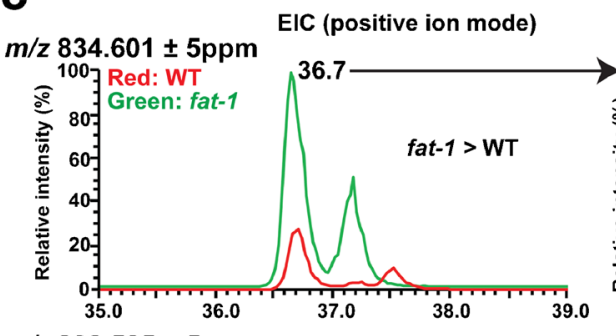

b
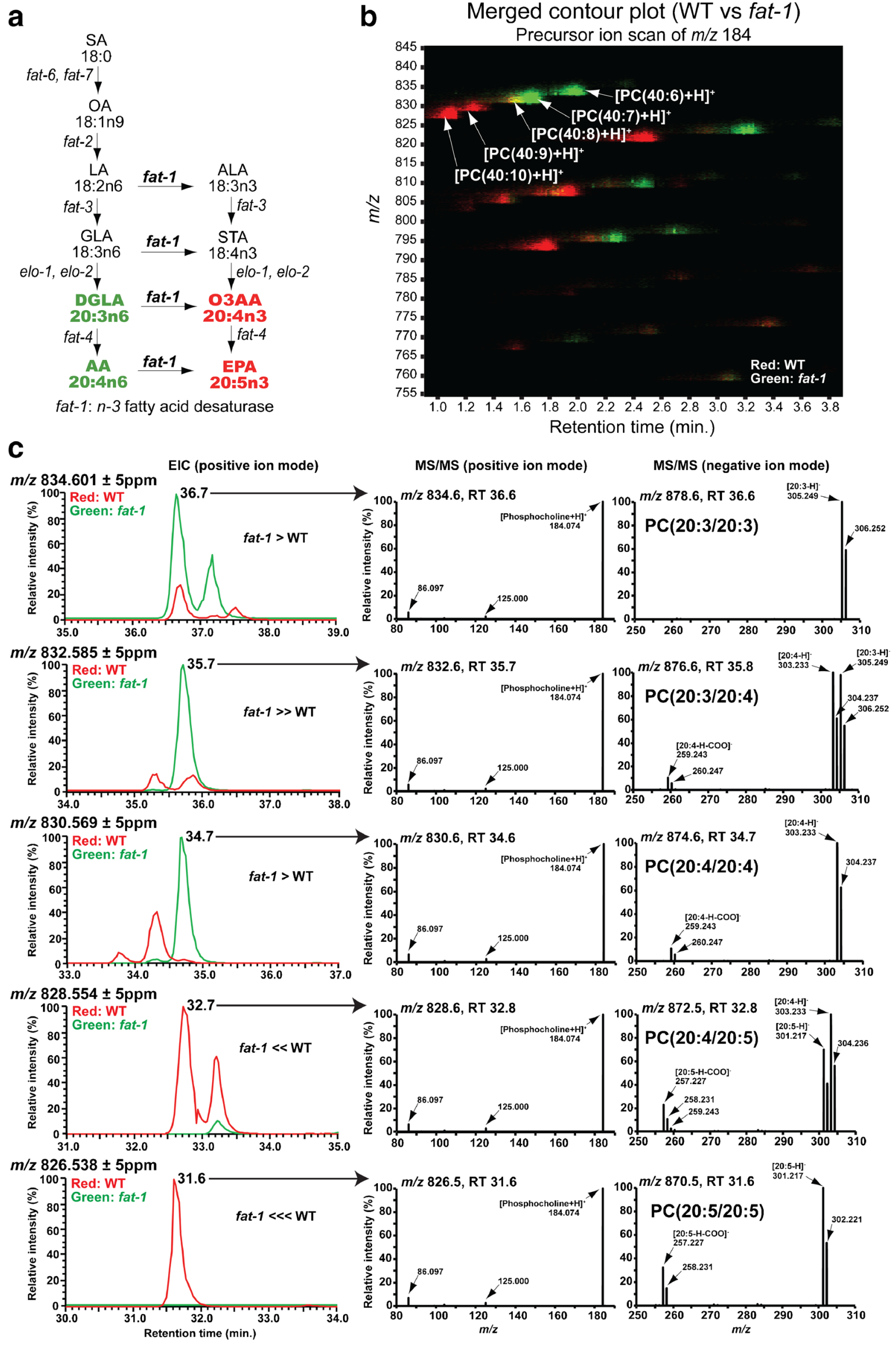
performed whole-body MALDI-IMS by applying the freezecracking method to both of the strains (Fig. 5a). The averaged mass spectra between $m / z$ 810-880 included five mass peaks with $\mathrm{m} / \mathrm{z}$ values of $864.5,866.5,868.5,870.5$, and 872.5 (Fig. 5b), which corresponded to the $\mathrm{K}^{+}$adducts $\left([\mathrm{M}+\mathrm{K}]^{+}\right)$ of the following PC species: $\operatorname{PC}(20: 5 / 20: 5), \operatorname{PC}(20: 4 / 20: 5)$, PC(20:4/20:4), PC(20:3/20:4), and PC(20:3/20:3), respectively. The IMS image of each PC species revealed similar PC composition differences between the wild-type and fat-1 mutants as were detected using LC-ESI-MS/MS (Fig. 5c). PC(20:5/20:5) and PC(20:4/20:5) had higher intensity signals in the wild-type (cyan-to-yellow colors in the thermal color scale) than in the fat-1 mutants (blue-to-cyan colors) (Fig. 5c). In contrast, $\mathrm{PC}(20: 4 / 20: 4), \operatorname{PC}(20: 3 / 20: 4)$, and $\mathrm{PC}(20: 3 / 20: 3)$ were more abundant in the fat-1 mutants (cyan-to-orange colors) than in the wild type (blue-to-cyan colors) (Fig. 5c). Semi-quantitative analyses clearly demonstrated the differences of the PC compositions between the wild-type and fat1 mutants $(n=6)$ (Fig. 5d), and their statistical values are given in Table 2. The signal intensities of $\operatorname{PC}(20: 5 / 20: 5)$ and $\operatorname{PC}(20: 4 / 20: 5)$ were higher in the wild-type than in the fat-1 mutants $(p<0.01$ and $p<0.05$, respectively, $t$ test), whereas the signal intensities of $\mathrm{PC}(20: 4 / 20: 4), \mathrm{PC}(20: 3 / 20: 4)$, and $\operatorname{PC}(20: 3 / 20: 3)$ were higher in the fat-1 mutants than in the wild type ( $p<0.01$ for all cases, $t$ test).

We also analyzed the wild-type and fat- 1 mutants in negative ion mode to test the effect of the fat-1 mutation on phospholipids composition besides PCs. The averaged mass spectra showed only a few peaks (Fig. 5e). The peaks at $m / z$ 758.4, $\mathrm{m} / \mathrm{z} 883.4$, and $\mathrm{m} / \mathrm{z} 885.4$ were selected to obtain images of those molecules with thermal color scale (Fig. 5f). Two molecules with $\mathrm{m} / \mathrm{z} 883.4$ and 885.4 were detected almost mutually exclusively in wild-type and fat-1 mutant (Fig. 5f): a molecule with $\mathrm{m} / \mathrm{z} 883.4$ was detected in wild type while a molecule with $\mathrm{m} / \mathrm{z} 885.4$ being detected in fat-1 mutants $(p<0.005, t$ test, $n=8)$ (Fig. $5 \mathrm{~g})$. No significant difference was detected for the molecule with $m / z 758.4$ ( $p>0.05, t$ test) (Fig. 5g). These results demonstrated that our whole-body MALDI-IMS technique was capable of being connected to genetics by analyzing individual strains.

\section{Discussion}

We presented a procedure for the direct analysis of the lipid compositions of the whole body of a well-established model organism, C. elegans, without requiring lipid extraction or target-specific labeling. We also showed that whole-body MALDI-IMS could offer analyses of molecular distribution in the nematode body and be combined with genetics. The key point of our protocol was to remove the exoskeletal cuticle from nematode bodies, aiming to efficiently generate cocrystals of biomolecules and matrices.
Fig. 5 Whole-body MALDI-IMS of genetically deficient nematodes. a Scanning electron microscopy images of freeze-cracked wild-type and fat 1 mutants. Scale bar: $25 \mu \mathrm{m}$. b Averaged mass spectra detected from freeze-cracked wild-type or fat-1 mutants in positive ion mode. Five mass peaks at $\mathrm{m} / \mathrm{z} 864.5, \mathrm{~m} / \mathrm{z} 866.5, \mathrm{~m} / \mathrm{z} 868.5, \mathrm{~m} / \mathrm{z} 870.5$, and $\mathrm{m} / \mathrm{z}$ 872.5 were selected to depict thermal color scale images. $\mathbf{c}$ Thermal color scale images of the five selected PC species. Scale bar: $250 \mu \mathrm{m}$. Color scale: deep blue, faint signal; red-purple, maximum signal. d Quantitative signal intensities of the PC species in the wild-type and fat- 1 mutants. The data are shown as plots for each nematode (diamonds; $n=6$ ) and mean (red line) $\pm \mathrm{SD}$ (blue lines). The $p$ values were calculated using a $t$ test. e Averaged mass spectra detected from freeze-cracked wild-type or fat-1 mutants in negative ion mode. Three mass peaks at $\mathrm{m} / \mathrm{z} 758.4, \mathrm{~m} / \mathrm{z} 883.4$, and $\mathrm{m} / \mathrm{z} 885.4$ were selected to depict thermal color scale images. $\mathbf{f}$ Thermal color scale images of the three selected mass peaks. Scale bar: $250 \mu \mathrm{m}$. Color scale: deep blue, faint signal; red-purple, maximum signal. g Quantitative signal intensities of the detected biomolecular species in the wild-type and fat- 1 mutants. The data are shown as plots for each nematode (diamonds; $n=8$ ) and mean (red line) $\pm \mathrm{SD}$ (blue lines). The $p$ values were calculated using a $t$ test

Before this study, two groups tried to directly analyze the components of nematodes without using extraction. One study detected some unidentified biomolecules in larval nematodes using time-of-flight secondary ion mass spectrometry (TOFSIMS) [41]. This work also combined whole-body imaging with genetics by comparing the molecular compositions of the wild-type and $d a f-2$ mutants [41]. TOF-SIMS was designed for the analysis of molecules present near the surface of specimens (within several nanometers) [42], and thus would require the removal or bypassing of the cuticle exoskeleton to analyze the intra-body composition. That study thus only detected molecules on the surface of the nematodes, although the cuticle of L1 larvae is thinner than that of adult nematodes [43]. Another study detected manganese, which is not a biomolecule, in larval nematodes using laser ablation-inductively coupled plasmamass spectrometry (LA-ICP-MS) [44]. LA-ICP-MS was designed for the analysis of elements such as metals, and uses a laser that is $>10$ times stronger $(>1 \mathrm{~mJ})[45,46]$ than those used in MALDI-IMS (a few hundred $\mu \mathrm{J}$ ). The high-powered laser degrades most organic compounds, although it enables the penetration of the cuticle layer and the ablation of intra-body materials. Furthermore, any degraded fragments of organic compounds would be completely destroyed within the ICP [47]. Thus, LA-ICP-MS is incapable of analyzing organic compounds. Our whole-body MALDI-IMS technique addressed these problems through co-crystallization of phospholipids with a matrix in the nematode body by removing the cuticle using freeze-cracking.

Our technique provided a lipidomic analysis of the PC species in genetically deficient mutant nematodes. The result of the comparative whole-body IMS between the wild-type and fat- 1 mutants is consistent with the fatty acid compositions reported previously [23-25]. The lower level of EPAcontaining PCs, $\mathrm{PC}(20: 5 / 20: 5)$ and $\mathrm{PC}(20: 4 / 20: 5)$, in fat-1 mutants is consistent with the finding that they lose EPA [24]. 

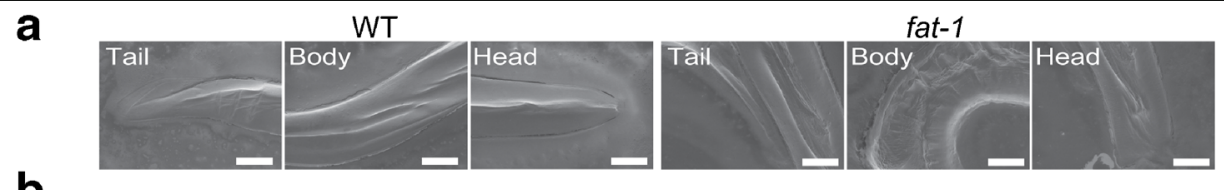

b
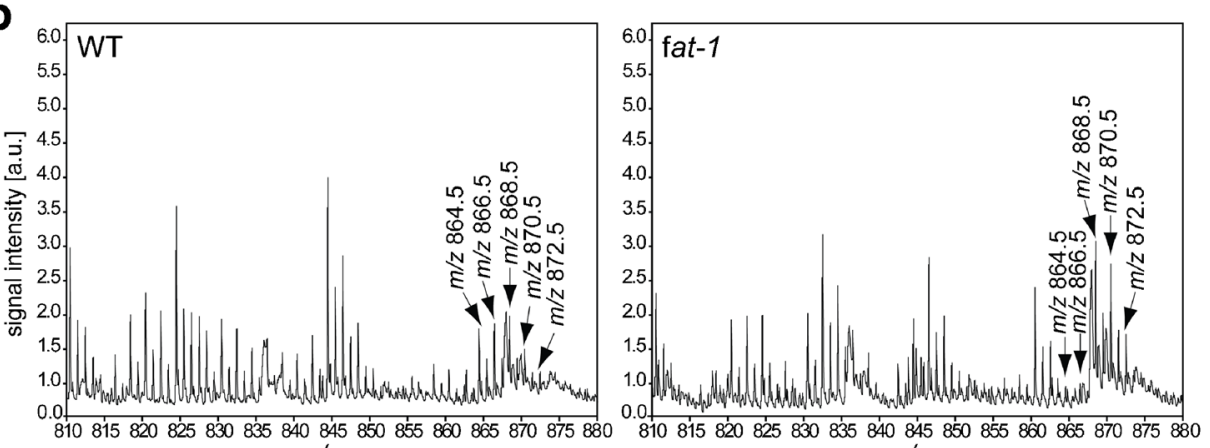

\section{C} 810815820825830835840845850855860865870875880810815820825830835840845850855860865870875880

$$
\text { WT }
$$
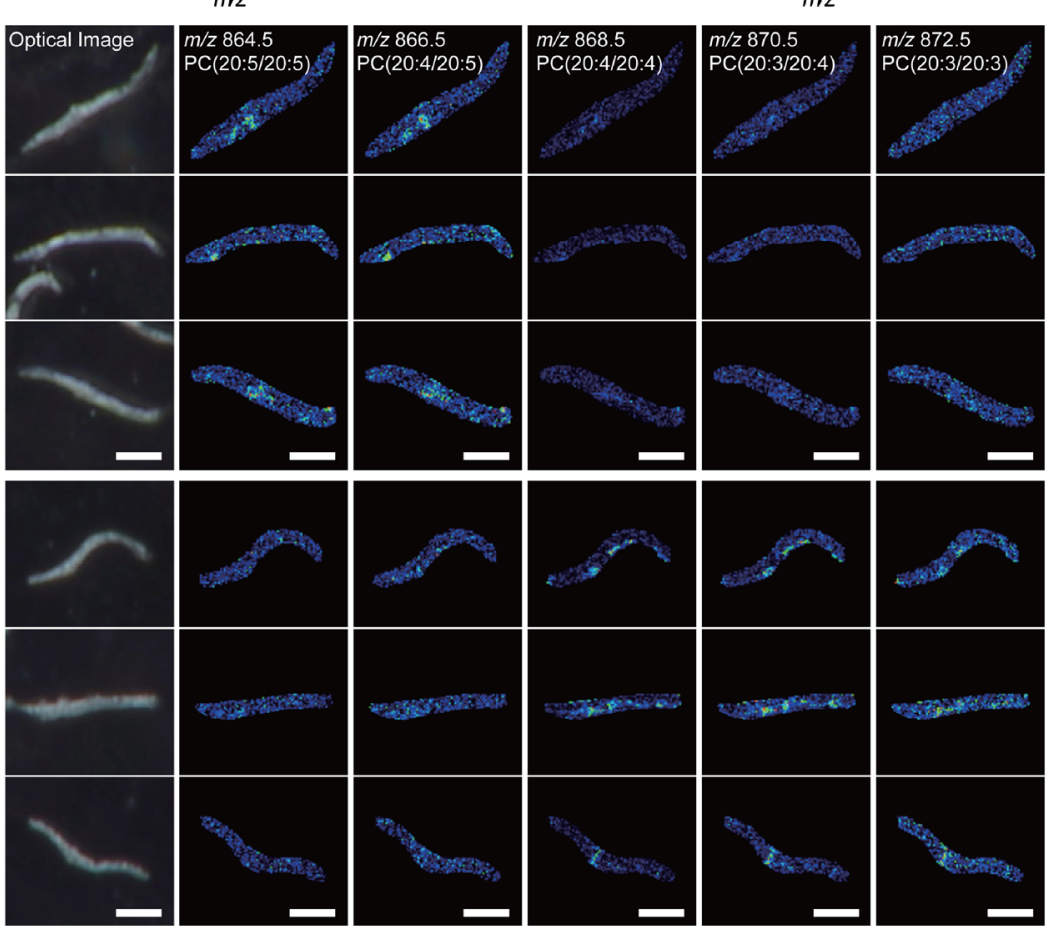

d
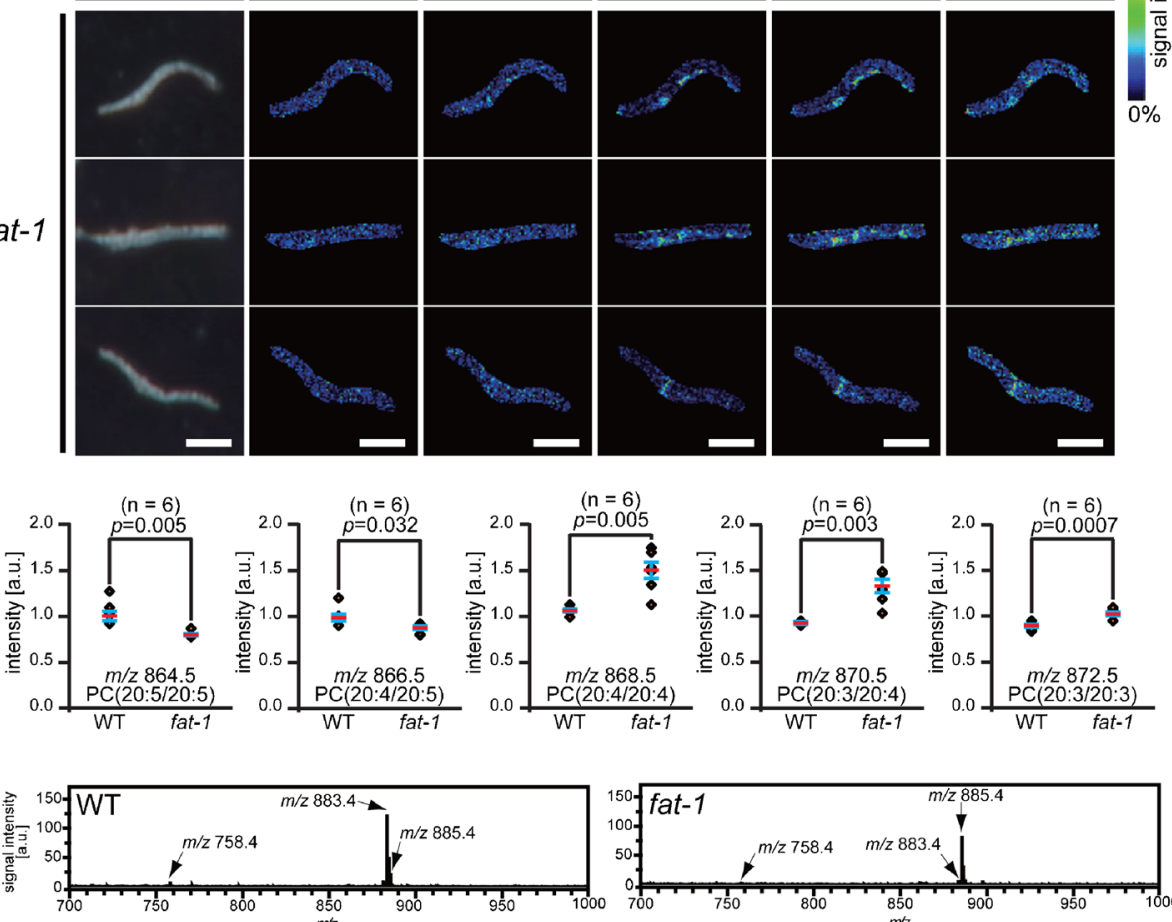

$(n=6)$
$\rho=0$

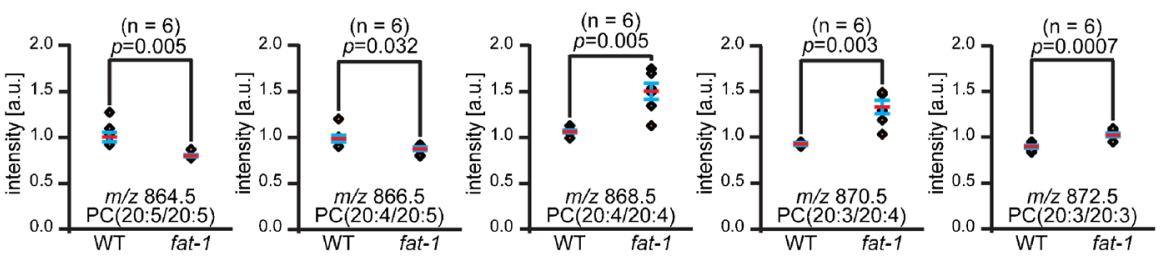

e
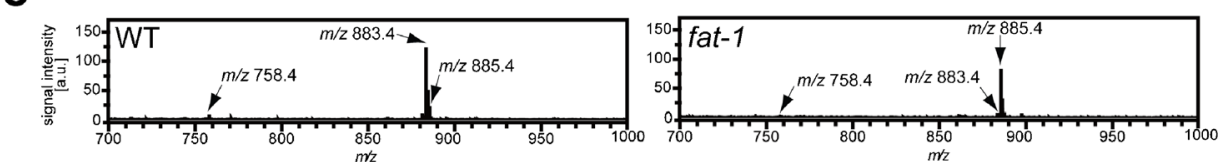

f
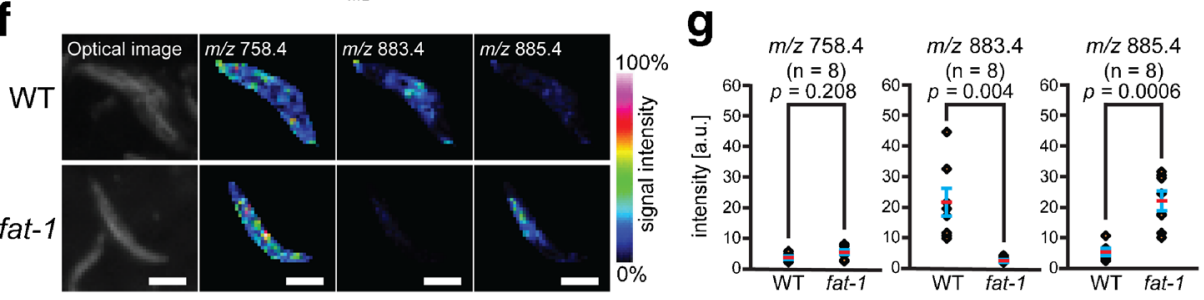
Table 2 Comparison of signal intensities in the wild-type and fat-1 mutants

\begin{tabular}{lllll}
\hline PC; $m / z$ & \multicolumn{2}{l}{ Mean signal intensity [a.u.] } & Number & $t$ test \\
\cline { 2 - 3 } & WT & fat-1 & WT/fat-1 & \\
\hline PC(20:5/20:5); 864.5 & $1.05 \pm 0.05$ & $0.81 \pm 0.001$ & $6 / 6$ & $<0.01$ \\
PC(20:4/20:5); 866.5 & $1.00 \pm 0.04$ & $0.87 \pm 0.002$ & $6 / 6$ & $<0.05$ \\
PC(20:4/20:4); 868.5 & $1.07 \pm 0.02$ & $1.50 \pm 0.09$ & $6 / 6$ & $<0.01$ \\
PC(20:3/20:4); 870.5 & $0.93 \pm 0.01$ & $1.34 \pm 0.008$ & $6 / 6$ & $<0.01$ \\
PC(20:3/20:3); 872.5 & $0.87 \pm 0.02$ & $1.03 \pm 0.03$ & $6 / 6$ & $<0.001$ \\
\hline
\end{tabular}

$W T$ wild type

The higher level of dihomo-gamma-linolenic acid (DGLA)containing PCs, PC(20:3/20:4) and PC(20:3/20:3), in fat- 1 mutants is consistent with the finding that DGLA is accumulated in them [24]. These results were also self-consistent with our own LC-ESI-MS/MS analyses. The higher level of PC(20:4/20:4) in fat-1 mutants is also consistent with the past finding that the amount of fatty acid (20:4) is higher in fat-1 mutants than the wild type [24]. Given that omega-3 arachidonic acid (O3AA; 20:4n3) was the major fatty acid (20:4) in the wild type and AA (20:4n6) was the sole fatty acid (20:4) in the fat-1 mutants [24], $\operatorname{PC}(20: 4 / 20: 4)$ in the wild type could be $\operatorname{PC}(20: 4 n 3 / 20: 4 n 3)$, whereas that in the fat-1 mutants could be $\mathrm{PC}(20: 4 \mathrm{n} 6 /$ 20:4n6). Notably, PC(20:4/20:4) was detected at different retention time in the wild-type $(33.7 \mathrm{~min})$ and the fat 1 mutants (34.7 $\mathrm{min})$. This highlights the caution required in data interpretation of MALDI-IMS in cases where molecules with identical $m / z$ values, but different structures, are compared, since the ionization efficiency in MALDI-IMS seems to differ based on molecular structure [48]. Some parts of our data might require the caution. The molecule with $m / z 868.5$ assigned as PC(20:4/ 20:4) in MALDI-IMS could be PC(20:3/20:5) in the wild type, and the molecule with $m / z 872.5$ assigned as PC(20:3/20:3) in MALDI-IMS could contain the fewer level of PC(20:2/20:4), based on the LC-ESI-MS/MS data. This structure-dependent complexity could be resolved more clearly when using IMS by means of ion mobility mass spectrometry in the future.

Our MALDI-IMS data in negative ion mode provided a clear difference of lipid composition between the wild-type and fat-1 mutants. The molecule with $\mathrm{m} / \mathrm{z} 883.4$ almost exclusively detected in the wild type is highly likely to be a PI containing EPA, PI(18:0/20:5), since $\mathrm{PI}(18: 0 / 20: 5)$ is the most major PI form in normal C. elegans [49, 50]. This idea is consistent with the knowledge that fat- 1 mutants lose EPA [24] and our findings that EPA-containing PCs were detected predominantly in the wild type. The molecule with $\mathrm{m} / \mathrm{z} 885.4$ is highly possible to be $\mathrm{PI}(18: 0 / 20: 4)$, which is rare in normal $C$. elegans $[49,50]$ while being normally detected and most major in mammalian tissues [51]. This idea is also consistent with the finding that AA is abnormally accumulated in fat-1 mutants [24] and our findings that PCs, $\mathrm{PC}(20: 3 / 20: 4)$ and $\mathrm{PC}(20: 4 / 20: 4)$, were detected almost exclusively in fat- 1 mutants.

Our technique could offer two additional benefits in the metabolomics analyses of nematodes. First, the technique provides spatial information regarding the uneven distribution of different biomolecules in individual nematode bodies. Rough sketches of molecular distribution could be determined in nematodes, as our IMS analyses combined with the matrix sublimation technique provided different mutually exclusive distribution of different molecules in a nematode body. Second, the technique could analyze multiple individuals in a single experiment. This has great potential for the comparison of individual-to-individual variations in molecular composition and metabolic responses to environmental stimuli. Taken together, our technique provides the potential to perform multi-dimensional omics analyses including the timedependent alteration of metabolite levels, at both the intraindividual and inter-individual levels.

Acknowledgments We would like to thank Drs. Naoko Goto-Inoue and Dan Nicolaescu for their critical reading of the manuscript and constructive comments. The nematode strains used in this work were donated by the Caenorhabditis Genetics Center, which is funded by the National Center for Research Resources, National Institutes of Health (NIH). This work was supported by JSPS KAKENHI for Scientific Research on Innovative Areas "Machinery of bioactive lipids in homeostasis and disease" (25116712) and for Kiban-B (25293044) (to M.S.), by JSPS KAKENHI for Kiban-C (25440082) (to Y.K.) and by the MEXT Project for Creation of Research Platforms and Sharing of Advanced Research Infrastructure. S.H. is receiving a scholarship from MEXT and formerly from JASSO and the Otsuka Toshimi Foundation.

Conflict of interest The authors declare that they have no conflicts of interest.

Open Access This article is distributed under the terms of the Creative Commons Attribution 4.0 International License (http:// creativecommons.org/licenses/by/4.0/), which permits unrestricted use, distribution, and reproduction in any medium, provided you give appropriate credit to the original author(s) and the source, provide a link to the Creative Commons license, and indicate if changes were made.

\section{References}

1. Hameed S, Sugiura Y, Kimura Y, Shrivas K, Setou M (2012) Nanoparticle-assisted laser desorption/ionization (nano-PALDI)based imaging mass spectrometry (IMS) and its application to brain sciences. In: Martin CR, Preedy VR, Hunter RJ (eds) Nanomedicine and the nervous system. Science Publishers, Boca Raton

2. Harada T, Yuba-Kubo A, Sugiura Y, Zaima N, Hayasaka T, GotoInoue N, Wakui M, Suematsu M, Takeshita K, Ogawa K, Yoshida Y, Setou M (2009) Visualization of volatile substances in different organelles with an atmospheric pressure mass microscope. Anal Chem 81:9153-9157 
3. Esquenazi E, Coates C, Simmons L, Gonzalez D, Gerwick W, Dorrestein P (2008) Visualizing the spatial distribution of secondary metabolites produced by marine cyanobacteria and sponges via MALDI-TOF imaging. Mol Biosyst 4:562-570

4. Thomas A, Charbonneu JL, Fournaise E, Chaurand P (2012) Sublimation of new matrix candidates for high spatial resolution imaging mass spectrometry of lipids: enhanced information in both positive and negative polarities after 1,5-diaminonapthalene deposition. Anal Chem 84:2048-2054

5. Eberlin L, Liu X, Ferreira C, Santagata S, Agar N, Cooks R (2011) Desorption electrospray ionization then MALDI mass spectrometry imaging of lipid and protein distributions in single tissue sections. Anal Chem 83:8366-8371

6. Caprioli R, Farmer T, Gile J (1997) Molecular imaging of biological samples: localization of peptides and proteins using MALDI-TOF MS. Anal Chem 69:4751-4760

7. Hayasaka T, Goto-Inoue N, Sugiura Y, Zaima N, Nakanishi H, Ohishi K, Nakanishi S, Naito T, Taguchi R, Setou M (2008) Matrix-assisted laser desorption/ionization quadrupole ion trap time-of-flight (MALDI-QIT-TOF)-based imaging mass spectrometry reveals a layered distribution of phospholipid molecular species in the mouse retina. Rapid Commun Mass Spectrom 22:3415-3426

8. Hirano H, Masaki N, Hayasaka T, Watanabe Y, Masumoto K, Nagata T, Katou F, Setou M (2014) Matrix-assisted laser desorption/ionization imaging mass spectrometry revealed traces of dental problem associated with dental structure. Anal Bioanal Chem 406:1355-1363

9. Matsumoto J, Sugiura Y, Yuki D, Hayasaka T, Goto-Inoue N, Zaima N, Kunii Y, Wada A, Yang Q, Nishiura K, Akatsu H, Hori A, Hashizume Y, Yamamoto T, Ikemoto K, Setou M, Niwa S (2011) Abnormal phospholipids distribution in the prefrontal cortex from a patient with schizophrenia revealed by matrix-assisted laser desorption/ionization imaging mass spectrometry. Anal Bioanal Chem 400:1933-1943

10. Uchiyama Y, Hayasaka T, Masaki N, Watanabe Y, Masumoto K, Nagata T, Katou F, Setou M (2014) Imaging mass spectrometry distinguished the cancer and stromal regions of oral squamous cell carcinoma by visualizing phosphatidylcholine (16:0/16:1) and phosphatidylcholine (18:1/20:4). Anal Bioanal Chem 406:13071316

11. Vrkoslav V, Muck A, Cvacka J, Svatos A (2010) MALDI imaging of neutral cuticular lipids in insects and plants. J Am Soc Mass Spectrom 21:220-231

12. Moree W, Phelan V, Wu C, Bandeira N, Cornett D, Duggan B, Dorrestein P (2012) Interkingdom metabolic transformations captured by microbial imaging mass spectrometry. Proc Natl Acad Sci U S A 109:13811-13816

13. Shroff R, Vergara F, Muck A, Svatos A, Gershenzon J (2008) Nonuniform distribution of glucosinolates in Arabidopsis thaliana leaves has important consequences for plant defense. Proc Natl Acad Sci U S A 105:6196-6201

14. Ferreira M, de Oliveira D, de Oliveira R, Allegretti S, Vercesi A, Catharino R (2014) Mass spectrometry imaging: a new vision in differentiating Schistosoma mansoni strains. J Mass Spectrom 49: $86-92$

15. Chansela P, Goto-Inoue N, Zaima N, Sroyraya M, Sobhon P, Setou M (2012) Visualization of neuropeptides in paraffin-embedded tissue sections of the central nervous system in the decapod crustacean, Penaeus monodon, by imaging mass spectrometry. Peptides 34:10-18

16. Niehoff AC, Kettling H, Pirkl A, Chiang YN, Dreisewerd K, Yew JY (2014) Analysis of Drosophila lipids by matrix-assisted laser desorption/ionization mass spectrometric imaging. Anal Chem 86: 11086-11092
17. Urban P, Chang C, Wu J, Chen Y (2011) Microscale MALDI imaging of outer-layer lipids in intact egg chambers from Drosophila melanogaster. Anal Chem 83:3918-3925

18. Brenner S (1974) The genetics of Caenorhabditis elegans. Genetics 77:71-94

19. Corsi AK (2006) A biochemist's guide to Caenorhabditis elegans. Anal Biochem 359:1-17

20. Fay D (2006) Genetic mapping and manipulation: chapter 1introduction and basics. WormBook 17:1-12. http://www. wormbook.org/chapters/www_introandbasics/introandbasics.html

21. Van Assche R, Broeckx V, Boonen K, Maes E, De Haes W, Schoofs L, Temmerman L (2015) Integrating -omics: systems biology as explored through C. elegans research. J Mol Biol. doi:10.1016/j. jmb.2015.03.015

22. O'Reilly LP, Luke CJ, Perlmutter DH, Silverman GA, Pak SC (2014) C. elegans in high-throughput drug discovery. Adv Drug Deliv Rev 69-70:247-253

23. Watts JL, Browse J (2006) Dietary manipulation implicates lipid signaling in the regulation of germ cell maintenance in C. elegans. Dev Biol 292:381-392

24. Watts J, Browse J (2002) Genetic dissection of polyunsaturated fatty acid synthesis in Caenorhabditis elegans. Proc Natl Acad Sci U S A 99:5854-5859

25. Satouchi K, Hirano K, Sakaguchi M, Takehara H, Matsuura F (1993) Phospholipids from the free-living nematode Caenorhabditis elegans. Lipids 28:837-840

26. Mahanti P, Bose N, Bethke A, Judkins JC, Wollam J, Dumas KJ, Zimmerman AM, Campbell SL, Hu PJ, Antebi A, Schroeder FC (2014) Comparative metabolomics reveals endogenous ligands of $\mathrm{DAF}^{-} 12$, a nuclear hormone receptor, regulating C. elegans development and lifespan. Cell Metab 19:73-83. doi:10.1016/j.cmet. 2013.11.024

27. Tanaka T, Ikita K, Ashida T, Motoyama Y, Yamaguchi Y, Satouchi K (1996) Effects of growth temperature on the fatty acid composition of the free living nematode Caenorhabditis elegans. Lipids 31: $1173-1178$

28. Wählby C, Conery AL, Bray MA, Kamentsky L, Larkins-Ford J, Sokolnicki KL, Veneskey M, Michaels K, Carpenter AE, O’Rourke EJ (2014) High- and low-throughput scoring of fat mass and body fat distribution in C. elegans. Methods 68:492-499

29. Page AP, Johnstone IL (2007) The cuticle. In: WormBook (ed) The C. elegans Research Community, WormBook, doi/10.1895/ wormbook.1.138.1, http://www.wormbook.org

30. Jun JH, Song Z, Liu Z, Nikolau BJ, Yeung ES, Lee YJ (2010) Highspatial and high-mass resolution imaging of surface metabolites of Arabidopsis thaliana by laser desorption-ionization mass spectrometry using colloidal silver. Anal Chem 82:3255-3265

31. Ohta I, Takaku Y, Suzuki H, Ishii D, Muranaka Y, Shimomura M, Hariyama T (2014) Dressing living organisms in a thin polymer membrane, the NanoSuit, for high-vacuum FE-SEM observation. Microscopy 63:295-300

32. Takaku Y, Suzuki H, Ohta I, Ishii D, Muranaka Y, Shimomura M, Hariyama T (2013) A thin polymer membrane, nano-suit, enhancing survival across the continuum between air and high vacuum. Proc Natl Acad Sci U S A 110:7631-7635

33. Wei Y, Zhang Y, Lin Y, Li L, Liu J, Wang Z, Xiong S, Zhao Z (2015) A uniform 2,5-dihydroxybenzoic acid layer as a matrix for MALDI-FTICR MS-based lipidomics. Analyst 140:1298-1305

34. Sugiura Y, Konishi Y, Zaima N, Kajihara S, Nakanishi H, Taguchi R, Setou M (2009) Visualization of the cell-selective distribution of PUFA-containing phosphatidylcholines in mouse brain by imaging mass spectrometry. J Lipid Res 50:1776-1788

35. Ellis SR, Brown SH, In Het Panhuis M, Blanksby SJ, Mitchell TW (2013) Surface analysis of lipids by mass spectrometry: more than just imaging. Prog Lipid Res 52:329-353 
36. Cerruti CD, Benabdellah F, Laprévote O, Touboul D, Brunelle A (2012) MALDI imaging and structural analysis of rat brain lipid negative ions with 9-aminoacridine matrix. Anal Chem 84:21642171

37. Fuchs B, Süss R, Schiller J (2010) An update of MALDI-TOF mass spectrometry in lipid research. Prog Lipid Res 49:450-475

38. Folch J, Lees M, Sloane Stanley GH (1957) A simple method for the isolation and purification of total lipides from animal tissues. $\mathrm{J}$ Biol Chem 226:497-509

39. Taguchi R, Houjou T, Nakanishi H, Yamazaki T, Ishida M, Imagawa M, Shimizu T (2005) Focused lipidomics by tandem mass spectrometry. J Chromatogr B Analyt Technol Biomed Life Sci 823:26-36

40. Murphy RC (2015) New developments in mass spectrometry No. 4. Tandem mass spectrometry of lipids: molecular analysis of complex lipids. Royal Society of Chemistry, Cambridge

41. Geier FM, Fearn S, Bundy JG, McPhail DS (2013) ToF-SIMS analysis of biomolecules in the model organism Caenorhabditis elegans. Surf Interface Anal 45:234-236

42. Vickerman JC, Briggs D (2013) Tof-SIMS: materials analysis by mass spectrometry, 2nd edn. IM Publications LLP, Chichester

43. Cox GN, Staprans S, Edgar RS (1981) The cuticle of Caenorhabditis elegans. II. Stage-specific changes in ultrastructure and protein composition during postembryonic development. Dev Biol 86:456-470

44. Brinkhaus SG, Bornhorst J, Chakraborty S, Wehe CA, Niehaus R, Reifschneider O, Aschner M, Karst U (2014) Elemental bioimaging of manganese uptake in C. elegans. Metallomics 6:617-621
45. Durrant SF, Ward NI (2005) Recent biological and environmental applications of laser ablation inductively coupled plasma mass spectrometry (LA-ICP-MS). J Anal At Spectrom 20:821-829

46. Hanć A, Komorowicz I, Iskra M, Majewski W, Barałkiewicz D (2011) Application of spectroscopic techniques: ICP-OES, LAICP-MS and chemometric methods for studying the relationships between trace elements in clinical samples from patients with atherosclerosis obliterans. Anal Bioanal Chem 399:3221-3231

47. Hill SJ (2006) Inductively coupled plasma spectrometry and its applications, 2nd edn. Wiley-Blackwell, Hoboken

48. Petkovic M, Schiller J, Müller M, Benard S, Reichl S, Arnold K, Arnhold J (2001) Detection of individual phospholipids in lipid mixtures by matrix-assisted laser desorption/ionization time-offlight mass spectrometry: phosphatidylcholine prevents the detection of further species. Anal Biochem 289:202-216

49. Lee HC, Inoue T, Imae R, Kono N, Shirae S, Matsuda S, GengyoAndo K, Mitani S, Arai H (2008) Caenorhabditis elegans mboa-7, a member of the MBOAT family, is required for selective incorporation of polyunsaturated fatty acids into phosphatidylinositol. Mol Biol Cell 19:1174-1184

50. Imae R, Inoue T, Kimura M, Kanamori T, Tomioka NH, KageNakadai E, Mitani S, Arai H (2010) Intracellular phospholipase A1 and acyltransferase, which are involved in Caenorhabditis elegans stem cell divisions, determine the $s n-1$ fatty acyl chain of phosphatidylinositol. Mol Biol Cell 21:3114-3124

51. Hicks AM, DeLong CJ, Thomas MJ, Samuel M, Cui Z (2006) Unique molecular signatures of glycerophospholipid species in different rat tissues analyzed by tandem mass spectrometry. Biochim Biophys Acta 1761:1022-1029 